\title{
II - Morbidade hospitalar por lesões e envenenamentos
}

\section{II - Hospital morbidity by lesions and poisonings}

\author{
Maria Lúcia Lebrão, Maria Helena P. de Mello Jorge e Ruy Laurenti \\ Departamento de Epidemiologia da Faculdade de Saúde Pública da Universidade de São Paulo. São \\ Paulo, SP - Brasil
}

\begin{abstract}
Resumo
As Autorizações de Internação Hospitalar (AIHs) hoje, no Brazil, representam aproximadamente $80 \%$ do total das internações do País e se tornaram de fácil acesso com a disseminação das bases de dados através de CD-ROM. Esse fato permite que se tenha um desenho, próximo do real, da morbidade que leva à hospitalização. Fazem parte dessa morbidade, as lesões e envenenamentos quer pela gravidade ou freqüência. Dada a sua importância, foram estudadas as internações de um mês do ano de 1994, por essas causas, segundo algumas características epidemiológicas e de serviço, como dados demográficos, diagnóstico, tempo de permanência e mortalidade. Verificou-se que as lesões e envenenamento têm sido responsáveis por 5 a $6 \%$ das internações do País, sendo preponderante no sexo masculino e, entre esses, foi a primeira causa de internações nos grupos etários de 15 a 29 anos. As mulheres somente os ultrapassam após os 65 anos. Quando analisadas segundo o tipo verifica-se que as fraturas são as mais frequientes $(37,5 \%)$ e dentre elas, as faturas dos membros são a quase totalidade. Dentre os ferimentos as localizações mais freqüentes são mão, antebraço, cabeça e pescoço. Considerando-se a idade e a maior preponderância no sexo masculino, pressupõe-se tratar de acidentes do trabalho ou do trânsito. Nas queimaduras chama a atenção a grande ocorrência nas crianças menores de 5 anos, o que leva os autores a lamentar a inexistência da informação sobre as causas desses acidentes, impedindo a possibilidade de ações de prevenção desses acontecimentos.
\end{abstract}

Inquéritos de morbidade. Ferimentos e lesões, diagnóstico. Envenenamento, diagnóstico.

\footnotetext{
Abstract

Today in Brazil the "H ospital Internment Authorizations" (AIH) represents about $80 \%$ of the total internments in the country and constitutes a data base of easy access available in CD-ROM. This high coverage allows one to draw a reasonable picture, close to reality, of the morbidity demanding hospitalization. A mong the causes there is the diagnostic category lesions and poisonings notew orthy for severity and frequency. Hospital internments by this diagnostic category occurring in a month in 1994 have been focused on. Some epidemiological and hospital use characteristics, such as demographic aspects, specific diagnostic category, lenght of stay and mortality are described. It was observed that lesions and poisonings accounted for $5 \%$ to $6 \%$ of all hospital internments in the country, being predominantly among men under 65 of age. This diagnostic category was the first cause of hospital in-
} 
ternments among men of from 15 to 29 years of age. Fractures were the most frequent specific diagnosis (about $37.5 \%$ ), almost all of them involving members (legs or arms). The injuries were mainly located in hands, arms, head and neck and occurred predominantly among young men, leading to the believe that they were due to work or traffic accidents. Regarding burns, the fregment occurrence among children under 5 years of age is worthy of note. The authors lament the lack of information as to their causes such as would enable appropriate preventive actions to be taken.

Morbidity surveys. Wounds and injuries, epidemiology. Poisoning, epidemiology.

\section{INTRO DU ÇÃO}

Embora as informações provenientes das estatísticas de hospitalizações sejam freqüentemente alvo de críticas, em razão de sua limitação quanto à abrangência, ou até mesmo pela sua qualidade, não resta dúvida de que elas podem ser bastante úteis para várias finalidades. Do ponto de vista administrativo e na avaliação da utilização de recursos financeiros são numerosas as possibilidades apresentadas pelas mesmas.

No Brasil, as hospitalizações pagas pelo sistema público de saúde são conhecidas através de um instrumento, preenchido nos hospitais, obrigatório para a internação dos pacientes e para o posterior recebimento dos pagamentos referentes a essas internações, que é a Autorização de Internação Hospitalar (AIH). Esse documento contém todas as informações relativas às internações, como dados demográficos, diagnósticos, procedimentos realizados e custos, o que permite o conhecimento do perfil da morbidade atendida por essa parcela do sistema e, de maneira importante, o cálculo dos custos das diferentes causas de internação.

Estima-se que o sistema AIH seja, atualmente, responsável por $80 \%$ da assistência médico-hospitalar prestada à população brasileira, representando cerca de 1.200.000 internações por mês (aproximadamente 14.000.000 internações/ano), em 6.380 unidades hospitalares, em 1991 (Levcovitz e Perei$\left.\mathrm{ra}^{7}, 1993\right)$.

Dessa forma, é possível ter-se um desenho quase completo da morbidade mais grave, a que leva à hospitalização. Nessa fração da morbidade, as "lesões e envenenamentos" têm um papel importante, seja pela sua gravidade, sua freqüência ou mesmo seu custo. No entanto, a qualidade dos registros médicos no Brasil deixa a desejar, como já se tem comentado amplamente nos meios acadêmicos ou na imprensa leiga (Lebrão ${ }^{6}, 1994$ e Veras $\left.^{18}, 1992\right)$. Não haveria porque ser diferente em relação às "lesões e envenenamentos". Além de todos os inconvenientes advindos dessa má qualidade da informação, neste caso há como agravante a questão da inexistência do diagnóstico secundário que, nas "lesões e envenenamentos", poderia significar o conhecimento das causas externas que motivaram essas lesões. Tal fato impede uma análise mais global das violências ocorridas.

Para os atendimentos de pronto-socorro não existe o preenchimento de AIH o que, especificamente quanto às lesões e envenenamentos, subestima a real importância da morbidade por essas causas.

O objetivo desta segunda parte, do presente trabalho, é conhecer a morbidade hospitalar por lesões e envenenamentos decorrentes de acidentes e violências, segundo algumas características epidemiológicas e de serviço consideradas importantes, no País.

\section{MATERIAL E MÉTO DO}

\section{Material de Estudo}

O material de estudo foi constituído por dados do Ministério da Saúde relativos às saídas hospitalares de hospitais próprios e conveniados do SUS para todo o País, analisadas a partir das AIHs.:

- de 1984 a 1993 (Ministério da Saúde ${ }^{9,10}, 1992,1995$ )

- de 1994, apresentados segundo algumas variáveis não trabalhadas para os anos anteriores (Ministério da Saúde ${ }^{10}$, 1995).

Além dessa análise global é feito o detalhamento das AIHs referentes ao mês de novembro de 1994, disponível em CD-ROM. Relativamente à representatividade desse mês, quando comparado aos meses restantes de anos anteriores, novembro não se mostrou mês atípico.

\section{Dados de População}

Foram utilizadas estimativas populacionais calculadas pelo método de Lagrange, a partir de dados oficiais do IBGE relativos aos censos de 1970, 1980 e 1991 (FIBGE $\left.{ }^{3,4,5}, 1973,1982,1993\right)$, conforme citado na Parte I do presente trabalho. 


\section{Metodologia}

Os totais de saídas hospitalares analisados referem-se aos dados captados por meio das AIHs, já referidas, salientando que:

1) para cada paciente é preenchida uma AIH que contém, entre outros, os seguintes dados, utilizados na presente análise:

- dados demográficos (idade, sexo)

- diagnóstico principal

- diagnóstico secundário

- procedimentos

- tempo de permanência

- tipo de saída

2) para pacientes com internação de longa permanência (ex. crônicos, de psiquiatria, de reabilitação) são emitidas novas AIH após 180 dias.

3) foi aceito como diagnóstico principal o assim apresentado na $\mathrm{AIH}$, teoricamente aquele que motivou a prestação do serviço.

A codificação desses diagnósticos foi feita segundo a Classificação Internacional de Doenças sendo que, durante todo o período analisado, esteve em vigor a $9^{\text {a }}$ Revisão (OMS/CID-9) ${ }^{14}$. Esses diagnósticos são mostrados segundo os capítulos da CID-9, acrescidos da "classificação suplementar de fatores que exercem influência sobre o estado de saúde e de oportunidades de contacto com serviços de saúde" (Código V).

Segundo regras internacionais (OMS/CID-9) ${ }^{14}$, os diagnósticos de causas externas a serem codificados nos registros de morbidade (tipo estatísticas hospitalares) devem usar as categorias do capítulo XVII (Lesões e envenenamentos) da Classificação Internacional de Doenças, dando, assim, ênfase à natureza da lesão. Portanto, esses diagnósticos são codificados como causa principal e o tipo de acidente ou violência que causou essa lesão, codificado pela "Classificação Suplementar de Causas Externas", será o diagnóstico secundário.

As AIHs em que, contrariamente às regras de codificação, constavam causas da Classificação Suplementar de Causas Externas (Código E), como diagnóstico principal, foram somadas às do Capítulo XVII - Lesões e envenenamentos, visto sua origem ser comum: lesões decorrentes de causas externas. Além disso, esse é o tratamento dado à questão, pelo DATASUS, órgão do Ministério da Saúde encarregado da apuração, análise e publicação desses dados.

Em relação às lesões, sua apresentação é feita com base na Lista Básica para Tabulação da CID-9.

Para a análise de algumas variáveis epidemiológicas, os casos trabalhados referiram-se a 73.357 AIHs do mês de novembro de 1994, pelas razões já expostas. Essas AIHs correspondem a:

1aㅡ 65.301 catalogadas no capítulo XVII da CID-9;

2 $\left.2^{\mathrm{a}}\right) 7.475$ casos em que, apesar de não constar o tipo de lesão/envenenamento que levou à internação, sabia-se existir um traumatismo, dado que, no diagnóstico principal constava algum tipo de acidente ou de violência. $3^{\text {a }) ~} 581$ casos em que as "lesões e envenenamentos" estavam mencionadas somente no diagnóstico secundário. Optou-se por incluir esses casos pelo fato de serem eles, provavelmente, resultantes de complicações ou efeitos tardios das "lesões e envenenamentos", representando, desse modo, uma internação ligada aos eventos em estudo.

\section{Medidas Utilizadas}

Foram as seguintes as medidas utilizadas:

taxa de internação $=\frac{\text { número de internações }}{\text { população }} \times 1.000$

tempo médio de permanência $=\frac{\text { número de dias de internação }}{\text { número de internações }}$

taxa de mortalidade $=\frac{\text { número de óbitos }}{\text { número de internações }} \times 100$

\section{RESU LTAD O S E DISC U SSÃO}

\section{- Evolução das H ospitalizações por Lesões e Envenenamentos}

As hospitalizações por "lesões e envenenamentos" no Brasil, na última década, têm sido responsáveis por 5 a $6 \%$ do total de internações do País. Porém, as taxas de internação por essa causa mostram uma tendência de aumento que, no período de 1984 a 1994 correspondeu a 60,6\% (Tabela 1). Como já foi comentado, as AIHs não permitem que se tenha a informação das causas externas desses acidentes e violências, dificultando o estabelecimento de algumas hipóteses que possam explicar esse aumento.

Nos Estados Unidos a proporção de internações por "lesões e envenenamentos" tem estado ao redor de 9,0\% do total, com ligeira tendência de queda. Com relação às taxas de internação por essas causas, os valores americanos são bem superiores aos verificados no Brasil (em 1981, 15,7; em 1988, 11,5 e, em 1991, 11,1 e 10,6 em 1993, todos medidos por 1.000 habitantes) ( Pokras $^{19}, 1989$; $\left.\mathrm{NCHS}^{11,12}, 1993,1995\right)$. Nos países nórdicos, onde esta causa está em terceiro ou quarto posto, o valor da taxa de internação variou de 13 a 20 por mil habitantes (Nomesco ${ }^{13}, 1992$ ).

Na França, em 1992, as "lesões e envenenamentos" estiveram em quinto lugar entre os motivos de internação, correspondendo a aproximadamente $8 \%$ do total (Com-Ruelle ${ }^{1}$, 1995). No Vale do Paraíba, em 1988, em análise de 41 hospitais gerais, essas causas foram responsáveis por $6,1 \%$, ficando em sexto posto (Lebrão $\left.{ }^{6}, 1994\right)$. Em alguns hospitais, dadas as suas características e localização, esse percentual pode chegar a atingir valores muito eleva- 
Tabela 1 - Hospitalizações por todas as causas e por lesões e envenenamentos ( $n^{\circ}$, \% e taxa de internação por 1.000 habitantes), Brasil, 1984/1994.

\begin{tabular}{|c|c|c|c|c|}
\hline \multirow{2}{*}{ Ano } & \multirow{2}{*}{$\begin{array}{c}\text { Todas } \\
\text { as causas }\end{array}$} & \multicolumn{3}{|c|}{ Causas externas } \\
\hline & & $\mathrm{N} 0$ & $\%$ & Taxa \\
\hline 1984 & 8.936 .644 & 443.643 & 5,0 & 3,4 \\
\hline 1985 & 7.874 .353 & 377.096 & 4,8 & 2,9 \\
\hline 1986 & 8.269 .189 & 401.676 & 4,9 & 3,0 \\
\hline 1987 & 9.611 .461 & 493.747 & 5,1 & 3,6 \\
\hline 1988 & 11.132 .957 & 595.507 & 5,4 & 4,3 \\
\hline 1989 & 11.431 .462 & 658.146 & 5,8 & 4,6 \\
\hline 1990 & 11.796 .360 & 684.523 & 5,8 & 4,7 \\
\hline 1991 & 13.568 .485 & 832.469 & 6,1 & 5,7 \\
\hline 1992 & 14.583 .191 & 884.587 & 6,1 & 5,9 \\
\hline 1993 & 14.829 .811 & $874.023^{*}$ & $5,9 *$ & $5,8^{*}$ \\
\hline 1994 & 14.698 .988 & 848.560 & 5,8 & 5,5 \\
\hline
\end{tabular}

* Com exceção do mês de novembro

dos, como é o caso do Hospital Municipal do Tatuapé, em São Paulo, em 1994, onde foi igual a 24,7\% (Paiva $\left.{ }^{15}, 1995\right)$.

No Brasil, esses números podem não ser tão elevados como nos outros países pelo fato de que aqui eles se referem apenas às internações pagas pelo Sistema Único de Saúde (SUS), não estando computados os casos atendidos em hospitais não conveniados com o SUS e, talvez, grande parte do atendimento realizado pelas redes estadual e municipal que, sabidamente, ao menos no Estado de São Paulo, é pouco faturado. Acresce ainda, conforme já comen- tado, que os atendimentos de pronto-socorro não são computados através das AIHs e é, a esse nível, que muitos dos casos de lesões e envenenamentos são atendidos e, até mesmo, resolvidos.

Ao se destacar, porém, o ano de 1994 (14.698.988 internações), pode-se ver, na Tabela 2, que as "lesões e envenenamentos", quando comparadas às demais causas, corresponderam a 5,8\% (848.560), ocupando o sétimo posto, sendo precedidas pelas "complicações da gravidez, parto e puerpério", "doenças do aparelho respiratório", "doenças do aparelho circulatório", "doenças infecciosas e parasitárias", "do-

Tabela 2 - Hospitalizações (número, tempo de permanência e taxa de mortalidade) segundo causas, Brasil, 1994.

\begin{tabular}{|c|c|c|c|c|}
\hline \multirow[b]{2}{*}{ Causas (CID-9) } & \multicolumn{2}{|c|}{ Internações } & \multirow[b]{2}{*}{ T.P. dias } & \multirow[b]{2}{*}{ TMP } \\
\hline & $\mathrm{N} 0$ & $\%$ & & \\
\hline D.Infecciosas e parasitárias & 1.445 .807 & 9,8 & 6 & 2,7 \\
\hline Neoplasias & 452.869 & 3,1 & 7 & 5,3 \\
\hline D.gl.end.,nutr., metab., & 418.531 & 2,9 & 7 & 4,8 \\
\hline D. sangue & 90.207 & 0,6 & 6 & 2,9 \\
\hline Transtornos mentais & 443.656 & 3,0 & 51 & 0,3 \\
\hline D. sist.nervoso e órg. sent. & 366.208 & 2,5 & 6 & 2,0 \\
\hline D. apar. circulatório & 1.518 .702 & 10,3 & 7 & 6,2 \\
\hline D. apar. respiratório & 2.441 .787 & 16,6 & 6 & 2,2 \\
\hline D. apar. digestivo & 1.067 .794 & 7,2 & 5 & 2,4 \\
\hline D. apar.geniturinário & 1.184 .397 & 8,0 & 4 & 0,7 \\
\hline Compl.grav.,parto,puerpério & 3.385 .380 & 23,0 & 2 & 0,0 \\
\hline D.pele e tec.conjuntivo & 152.441 & 1,0 & 6 & 0,8 \\
\hline D.sist.osteomusc.e tec.conj. & 318.164 & 2,2 & 5 & 0,3 \\
\hline Anomalias congênitas & 66.737 & 0,5 & 6 & 3,1 \\
\hline Alg.afec.per.perinatal & 201.945 & 1,4 & 8 & 6,6 \\
\hline Sint.,sin. e afec. maldefinidas & 244.869 & 1,7 & 4 & 3,7 \\
\hline Lesões e envenenamentos & 848.560 & 5,8 & 5 & 2,2 \\
\hline Classif. suplem. “ V” & 50.934 & 0,4 & 9 & 2,1 \\
\hline Total & 14.698 .988 & 100,0 & 6 & 2,2 \\
\hline
\end{tabular}

Fonte: Ministério da Saúde, 1995.

TP- Tempo de permanência

TMH - Taxa de mortalidade hospitalar (por 100 internações). 
enças do aparelho geniturinário" e "doenças do aparelho digestivo" (Figura 1).

Dentre as "doenças do aparelho respiratório", que foram responsáveis por $16,6 \%$ do total das internações, quase a metade, $7,8 \%$, foi devida a "pneumonias" e $4,1 \%$ a "doenças pulmonares obstrutivas crô-

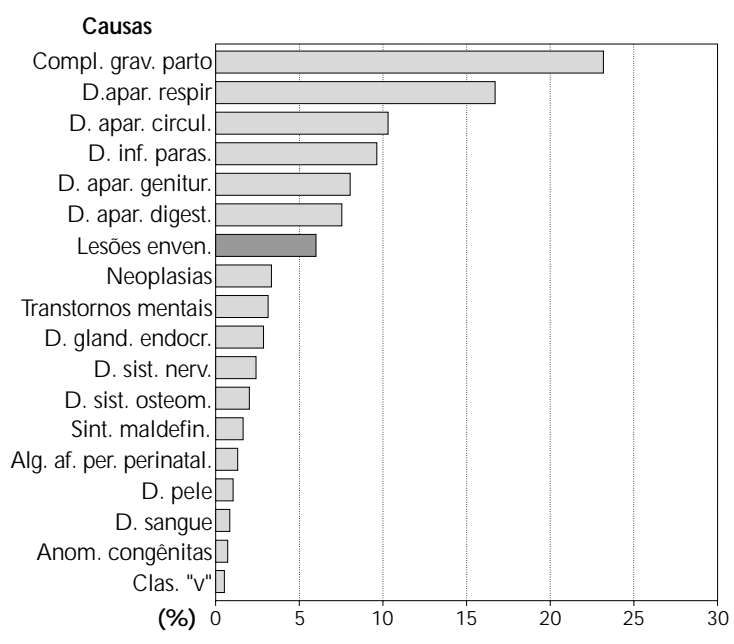

Figura 1 - Hospitalizações segundo causas de doenças, Brasil, 1994. nicas", com ênfase em "asma". Dentre as "doenças do aparelho circulatório", são relevantes as "insuficiências cardíacas" e, entre as "doenças infecciosas e parasitárias", as "diarréias" ocupam praticamente dois terços do total.

A ordenação dessas causas foi equivalente à verificada no Estado de São Paulo, em 1980, em pesquisa realizada por meio dos Boletins da Secretaria Estadual de Saúde (Ferreira e Faria ${ }^{2}, 1986$ ), bem como, com pequenas alterações, à de Ribeirão Preto no início da década de 70, com dados do Centro de Processamento de Dados Hospitalares (Yazlle Rocha ${ }^{19}, 1980$ ).

No entanto, quando se excluem as "complicações da gravidez, parto e puerpério", pelo fato de estarem concentradas no sexo feminino e na faixa etária fértil, o que levaria certamente a resultados distorcidos, verifica-se que as "lesões e envenenamentos" passam a ser responsáveis por 7,5\% do total das internações.

A importância das lesões e envenenamentos pode ser evidenciada pelo fato de serem elas a primeira causa de internações nas idades de 15 a 19 anos $(24,7 \%)$ e de 20 a 29 anos $(22,6 \%)$, no sexo masculino. Também nas idades de 30 a 39 anos essas causas são relevantes $(16,2 \%)$, estando abaixo apenas dos transtornos mentais.

Tabela 3 - H ospitalizações por lesões e envenenamentos segundo sexo e idade, Brasil, novembro de 1994.

\begin{tabular}{|c|c|c|c|c|c|c|}
\hline \multirow[t]{2}{*}{ Idade } & \multicolumn{2}{|c|}{ Masculino } & \multicolumn{2}{|c|}{ Feminino } & \multicolumn{2}{|c|}{ Total } \\
\hline & $\bar{N} 0$ & $\%$ & No 0 & $\%$ & $\mathrm{~N} 0$ & $\%$ \\
\hline-5 & 3.345 & 4,6 & 2.514 & 3,4 & 5.859 & 8,0 \\
\hline 5 a 9 & 4.115 & 5,6 & 2.093 & 2,9 & 6.208 & 8,5 \\
\hline 10 a 14 & 4.413 & 6,1 & 1.694 & 2,3 & 6.007 & 8,2 \\
\hline Sutotal & 11.773 & 16,1 & 6.301 & 8,6 & 18.074 & 24,8 \\
\hline 15 a 19 & 4.958 & 6,8 & 1.847 & 2,5 & 6.805 & 9,3 \\
\hline 20 a 24 & 5.841 & 8,0 & 1.868 & 2,6 & 7.709 & 10,6 \\
\hline 25 a 29 & 5.208 & 7,1 & 1.709 & 2,3 & 6.917 & 9,5 \\
\hline 30 a 34 & 4.469 & 6,1 & 1.628 & 2,2 & 6.097 & 8,4 \\
\hline 35 a 39 & 3.676 & 5,0 & 1.423 & 2,0 & 5.099 & 7,0 \\
\hline 40 a 44 & 3.068 & 4,2 & 1.302 & 1,8 & 4.370 & 6,0 \\
\hline Subtotal & 27.220 & 37,3 & 9.777 & 13,4 & 36.997 & 50,7 \\
\hline 45 a 49 & 2.298 & 3,2 & 1.015 & 1,4 & 3.315 & 4,5 \\
\hline 50 a 54 & 1.870 & 2,6 & 1.001 & 1,4 & 2.871 & 3,9 \\
\hline 55 a 59 & 1.492 & 2,0 & 952 & 1,3 & 2.444 & 3,4 \\
\hline 60 a 64 & 1.313 & 1,8 & 977 & 1,3 & 2.290 & 3,1 \\
\hline Subtotal & 6.973 & 9,6 & 3.945 & 5,4 & 10.918 & 15,0 \\
\hline 65 a 69 & 1.025 & 1,4 & 928 & 1,3 & 1.953 & 2,7 \\
\hline 70 a 74 & 774 & 1,1 & 900 & 1,2 & 1.674 & 2,3 \\
\hline 75 a 79 & 573 & 0,8 & 781 & 1,1 & 1.354 & 1,9 \\
\hline 80 e + & 708 & 1,0 & 1.231 & 1,7 & 1.939 & 2,7 \\
\hline Subtotal & 3.080 & 4,2 & 3.840 & 5,3 & 6.920 & 9,5 \\
\hline Total & 49.046 & 67,3 & 23.863 & 32,7 & 72.909 & 100,0 \\
\hline
\end{tabular}

N ota: Em 448 casos não foi possível determinar o sexo e/ou idade. 
Com relação ao tempo de permanência, a média foi de 6 dias, levando em consideração todas as causas em conjunto. Excetuando-se os "transtornos mentais", cuja média foi de 51 dias, em todos os demais grupos essas médias variaram entre 2 e 9 dias, respectivamente, para "gravidez, parto e puerpério" e "contatos com o serviço de saúde" (código V). Nesse último grupo, têm importância os "cuidados de seguimentos de ortopedia" e "observação e avaliação de condições suspeitas: mentais", o que explica a alta permanência. Nas lesões e envenenamentos, a média de permanência foi de 5 dias, bastante próxima, portanto, da média geral.

A taxa de mortalidade hospitalar (TMH), para todos os tipos de internação, variou entre 0,04 e 6,6\%. As maiores taxas corresponderam às "afecções originadas no período perinatal" $(6,6 \%)$ e "doenças do aparelho circulatório" (6,2\%), estando as "lesões e envenenamentos" no décimo posto, com 2,2 óbitos por cem internações. Na pesquisa realizada no Estado de São Paulo, (Ferreira e Faria², 1980) as causas perinatais, neoplasmas e doenças do aparelho circulatório foram os grupos mais importantes. Esse indicador, no geral, não tem grande valor pois é necessário conhecer o perfil das internações quanto à gravidade do caso, idade do paciente, existência ou não de diagnóstico associado e outros. No entanto, seu cálculo segundo as causas já é um avanço na sua capacidade de discriminação. Dessa forma, pode-se dizer, com alguma cautela, que, para as causas externas, o valor de $2,2 \%$ não é excessivo.

\section{H ospitalizações Segundo Sexo e Idade}

A Tabela 3, que mostra a distribuição das internações segundo sexo e idade, permite ver a preponderância do sexo masculino sobre o feminino $(67,3 \%$ e $32,7 \%$ ), ou seja, o dobro de homens em relação às mulheres, quando se analisa o total. Porém, nos diferentes grupos de idade esse comportamento se altera. Assim, entre os menores de 15 anos a relação masculino/feminino é 1,9; no grupo de 15 a 44 é de 2,8 , sendo o máximo $(3,1)$ no grupo de 20 a 29 anos; na faixa de 45 a 64 anos é de 1,8 e, no grupo de maiores de 65 anos esse valor se inverte, passando de 4,2\% de internações nos homens para $5,3 \%$ de internações no sexo feminino, correspondendo, portanto, a 1,2 hospitalizações de mulher para cada uma feita entre os homens.

Essa maior freqüência feminina nas faixas mais elevadas de idade, talvez se deva à maior sobrevida da mulher e às fraturas de fêmur, mais freqüentes no sexo feminino. Na análise das internações segundo o tipo da lesão será possível ver as diferenças nessa distribuição.

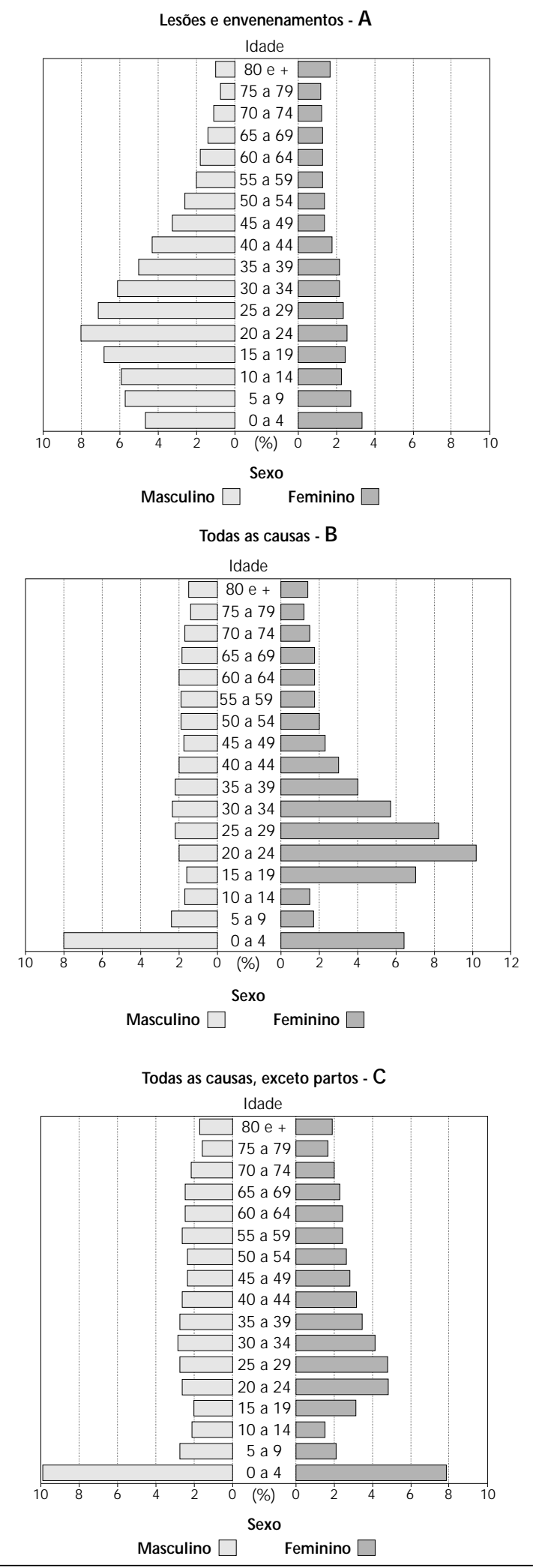

Figura 2 (a, b, c) - Hospitalizações por “lesões e envenenamentos", por todas as causas e por todas as causas exceto "partos", segundo sexo e idade, Brasil, novembro/1994. 
Quanto às idades, verifica-se a preponderância do adolescente e adulto jovem: $50 \%$ dos internados têm de 15 a 44 anos, sendo a faixa etária de 20 a 24 anos a de maior freqüência de internações, quando vista isoladamente.

Pode-se ter melhor visualização desse fato na Figura 2.

Ainda na Figura 2, para efeito de comparação, pode ser vista a distribuição das internações segundo sexo e idade para todas as causas e para o conjunto destas com exclusão dos partos.

Quanto às taxas de internação por lesões e envenenamentos segundo sexo, o valor foi de 7,7 por mil homens, representando pouco mais que o dobro da taxa verificada para as mulheres $(3,7$ por mil) (Tabela 4). Essa predominância ocorre também em outros países, como os Estados Unidos que, em 1993, embora com diferença bem menor, apresentaram coeficientes de 11,2 para o sexo masculino e 10,0 para o feminino (NCHS ${ }^{12}$, 1995). A distribuição nas faixas etárias mostra que as relações mais elevadas entre as taxas estão nos grupos de 20 a 29 anos, seguido do 30 a 39 e 15 a 19 anos. Nas mulheres de 70 anos e mais a taxa, equivalente ao dobro da faixa etária anterior, passa a ser maior que a dos homens.

\section{- Lesões e Envenenamentos Segundo Tipo}

A Tabela 5 mostra a participação de cada tipo de lesão no total das internações por esse grupo de causas. Somente foi possível detalhar o tipo de lesão
Tabela 4 - Taxa de internação* por "lesões e envenenamentos" segundo sexo e idade e relação Masculino/Feminino (M/F), Brasil, novembro de 1994.

\begin{tabular}{lccc}
\hline Idade & Masculino & Feminino & Relação M/F \\
\hline 0 a 4 & 4,9 & 3,8 & 1,3 \\
5 a 9 & 5,3 & 2,8 & 1,9 \\
10 a 14 & 5,9 & 2,3 & 2,6 \\
15 a 19 & 8,0 & 2,9 & 2,8 \\
20 a 29 & 9,9 & 3,1 & 3,2 \\
30 a 39 & 8,9 & 3,1 & 2,9 \\
40 a 49 & 8,7 & 3,6 & 2,4 \\
50 a 59 & 8,8 & 4,5 & 2,0 \\
60 a 69 & 8,6 & 6,2 & 1,4 \\
70 e mais & 11,8 & 13,1 & 0,9 \\
\hline Total & 7,7 & 3,7 & 2,1 \\
\hline
\end{tabular}

* Por 1.000 habitantes do sexo/idade considerados

que originou a internação em $65.301(89,0 \%)$ das hospitalizações por "lesões e envenenamentos", no mês de novembro.

As fraturas foram as responsáveis pelo maior número de internações $(37,5 \%)$, sendo que, dentre elas, as dos membros respondem pela quase totalidade $(84,5 \%)$ desse grupo. Em segundo lugar apareceram os "ferimentos e as lesões traumáticas dos vasos sangüíneos" $(17,2 \%)$ onde surgem, com mais da metade dos casos, os ferimentos de cabeça, pescoço e tronco. Com valor praticamente igual estão as lesões intracranianas e internas (16,9\%), das quais $84,0 \%$ são traumatismos intracranianos (Figura 3). As taxas de internação, segundo tipos de lesão, mos-

Tabela 5 - Hospitalizações por “lesões e envenenamentos" (№, \% e coef.*) segundo tipo da lesão, Brasil, novembro de 1994.

\begin{tabular}{lrrr}
\hline Lesões & $\mathrm{N} 0$ & $\%$ & Coef.* \\
\hline Fraturas & 24.460 & 37,5 & 1,9 \\
- crânio & 2.679 & 4,1 & \\
- pescoço e tronco & 1.086 & 1,7 & 0,2 \\
- membros superiores & 10.268 & 15,7 & 0,9 \\
Luxações/entorses/contusões & 10.427 & 16,0 & 0,9 \\
Lesões intracranianas e internas & 2.493 & 3,8 & \\
- traumat. intracranianos & 11.068 & 16,9 & \\
Ferimentos e les. traum.vasos sang. & 9.289 & 14,2 & 0,03 \\
- fer. cabeça, pesc. e tronco & 11.199 & 9,3 & 0,3 \\
- fer.membros superiores & 6.043 & 3,4 & 0,4 \\
Efeitos de corpos estranhos & 2.207 & 4,4 & 0,6 \\
Queimaduras & 2.888 & 5,3 & 0,2 \\
- queimaduras múltiplas & 375 & 3,5 & 0,3 \\
Envenenamentos e efeitos tóxicos & 3.465 & 7,2 & 0,0 \\
Complic. cuidados médicos & 2.276 & 3,7 & \\
Outras lesões & 4.714 & 6,7 & 1,1 \\
Efeitos tardios & 2.407 & 100,0 & \\
\hline Total & 4.386 & & \\
\hline
\end{tabular}

* por 1.000 habitantes 
tram, para as fraturas e lesões intracranianas, valores mais elevados que os dos Estados Unidos em 1993, respectivamente, 1,2 e 0,6 NCHS $^{12}$, 1995).

Na Figura 4 estão distribuídos alguns tipos de "lesões e envenenamentos" segundo sexo e idade. Da mesma maneira como foi visto para as "lesões e envenenamentos" como um todo, também quando se

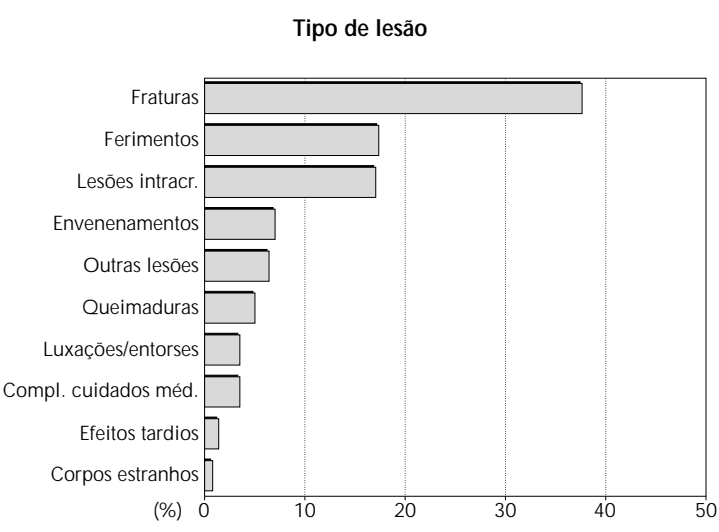

Figura 3 - Hospitalizações por "lesões e envenenamentos" (\%) segundo tipo de lesão, Brasil, novembro de 1994.

detalham essas internações segundo tipo, permanece a preponderância do sexo masculino, embora com valores diferentes (lesões intracranianas: 2,7; fraturas: 2,2; ferimentos: 2,0; queimaduras e envenenamentos: 1,4).

Nas fraturas chama a atenção a pouca representatividade dos menores de 5 anos, havendo concentração no lado masculino nas faixas etárias do préescolar, adolescente e adulto jovem. Percebe-se, ainda, a pequena participação das mulheres em todas as idades, com exceção das faixas de 5 a 9, de 10 a 14 anos e de 80 anos e mais, notando-se, ainda, uma discreta predominância feminina nos maiores de 60 anos.

Em relação às lesões intracranianas há que se notar, e estranhar, a grande participação dos menores de 5 anos, quase igualmente meninos e meninas. Pode se dizer, ainda, que estudos de mortalidade mostram que, do total de óbitos por "lesões intracranianas", 39,0\% decorreram de acidentes de trânsito e $24,2 \%$ de homicídios. Contrariamente, quando se leva em conta os traumatismos internos, verifica-se que $65,9 \%$ resultaram de homicídios, enquanto que apenas $16,8 \%$ são de acidentes de trânsito (Santo ${ }^{17}$, 1988).

Também nos ferimentos predomina o sexo masculino. Observando-se suas localizações mais freqüentes (mão e antebraço ou cabeça e pescoço) e a idade em que ocorrem supõe-se tratar, respectivamente, de acidentes do trabalho e acidentes de trânsito ou queda.

Nas queimaduras, chama a atenção sua ocorrência na faixa etária dos menores de 5 anos $(17,1 \%$ nos meninos e $13,4 \%$ nas meninas). Se se pudesse dispor, nas AIHs, da informação sobre os tipos de acidentes/violências que ocasionaram as internações, esse fato se constituiria em importante subsídio para programas de prevenção dessas causas. Nos estudos de mortalidade verifica-se que o grupo etário 1 a 4 anos é o que prepondera nas queimaduras por objetos ou substâncias quentes, principalmente líquidos. Em pesquisa realizada em São Paulo pôde se constatar que os acidentes que levaram a óbito, nessa faixa etária, se constituíram em acidentes domésticos, na grande maioria dos casos, decorrendo de queda de panela com líquido quente sobre a vítima (Mello Jorge $^{8}, 1988$ )

Quanto às internações por envenenamentos, foi possível verificar que esse é o grupo em que as mulheres têm maior participação, embora não preponderante. Esse fato se dá às custas das "intoxicações por drogas e medicamentos", que é muito mais importante no sexo feminino. Lebrão ${ }^{6}$ (1994), em trabalho a respeito da evolução da morbidade hospitalar no tempo, tendo como exemplo uma região do Estado de São Paulo, encontrou perfis de alguns tipos de lesões semelhantes aos vistos no presente trabalho. Porém, em relação aos envenenamentos, comportamento como o do Brasil foi encontrado somente nos dados de 1975 , tendo a participação da mulher sido bastante reduzida no intervalo considerado de 13 anos. Isso foi atribuído à eficácia das medidas de controle, exercidas pelo Estado, sobre a venda de entorpecentes e tranqüilizantes (Lebrão $\left.{ }^{6}, 1994\right)$. Entre os homens foi mais freqüente o "efeito tóxico do álcool”, mais especificamente álcool etílico, casos que talvez estejam erroneamente codificados, pois essas internações devem se referir às "intoxicações agudas pelo álcool". Estes, se codificados corretamente, deveriam estar nos "abusos de droga sem dependência" que, por sua vez, segundo disposição internacional (CID-9), são alocados no grupo dos "transtornos mentais".

A Tabela 6 mostra a distribuição dos tipos de lesão dentro de cada grupo etário. Embora, como já foi visto, as fraturas se constituam no tipo mais importante de lesões, é no grupo etário de 65 e mais anos onde elas aparecem com maior peso. Nesta faixa, as fraturas de membros inferiores representam $70 \%$, tanto nas internações masculinas quanto nas femininas. Ao se calcular os coeficientes de internação das fraturas 

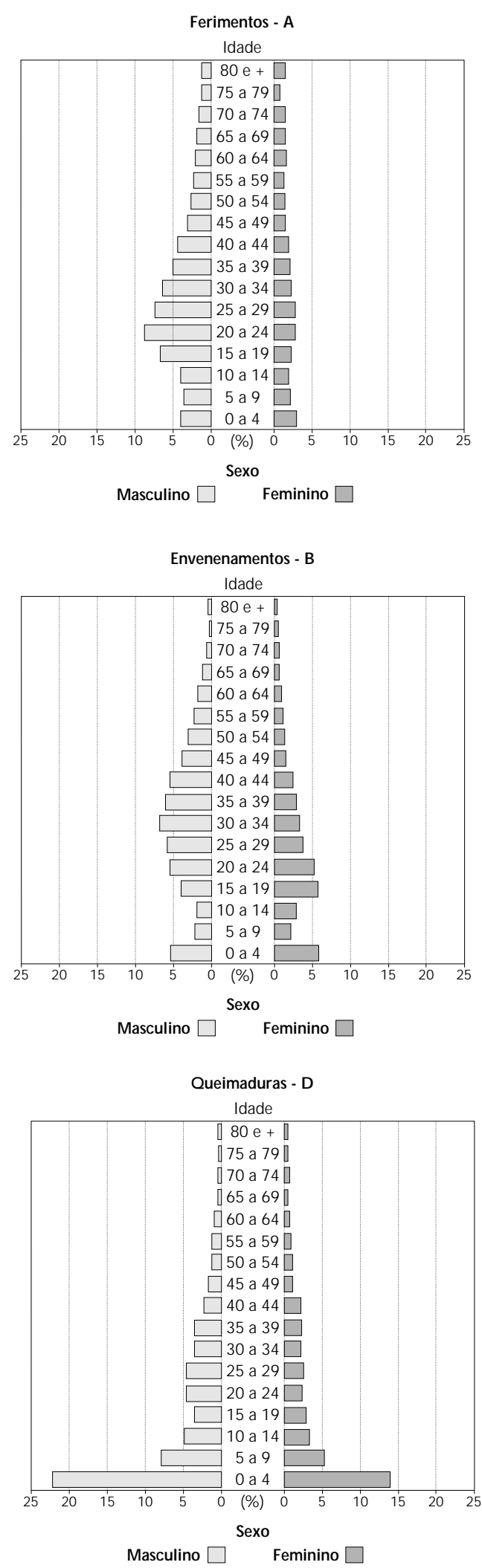

de fêmur, segundo o sexo, verifica-se que a maior proporção de mulheres nas internações por essa causa, após os 70 anos, tem sua base biológica, não dependendo apenas da maior sobrevivência feminina, pois os coeficientes são 3,1 e 5,7 por mil homens ou mulheres, respectivamente. As lesões intracranianas e internas, por sua vez, têm pouca importância nesse grupo etário, aparecendo nas demais idades, com valores próximos, com ênfase nos menores de 15 anos. Em relação às queimaduras, elas são, também, mais significativas para o grupo de menores de 15 anos do que para qualquer outro. Ao se detalhar as idades dentro dessa faixa, nota-se que elas se concentram nos menores de 5 anos, conforme se vê na Figura 3 e, mais intensamente ainda, nas idades de 1 e 2 anos, que so-

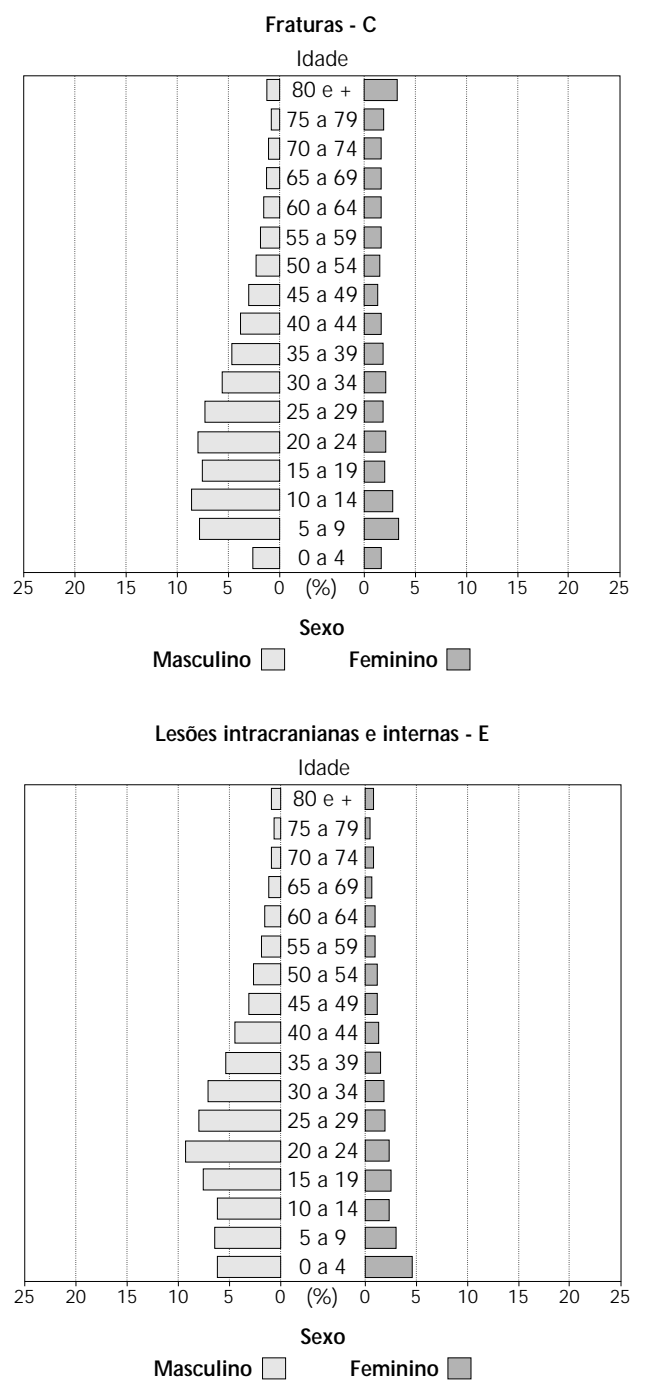

Figura 4 (a, b, c, d e e) - Hospitalizações por alguns tipos de "lesões e envenenamentos" segundo idade e sexo, Brasil, novembro de 1994. 
Tabela 6 - Hospitalizações por "lesões e envenenamentos" ( $N$ o e \%) segundo tipo da lesão e idade, Brasil, novembro de 1994.

\begin{tabular}{|c|c|c|c|c|c|c|c|}
\hline Lesões & & $<15$ & 15 a 44 & 45 a 64 & $65 \mathrm{e}+$ & $\operatorname{lgn}$. & Total \\
\hline Fraturas & $\begin{array}{l}\text { No } \\
\%\end{array}$ & $\begin{array}{c}6.434 \\
39,1\end{array}$ & $\begin{array}{c}11.379 \\
35,2\end{array}$ & $\begin{array}{l}3.542 \\
36,5\end{array}$ & $\begin{array}{c}2.994 \\
47,1\end{array}$ & $\begin{array}{c}111 \\
26,7\end{array}$ & $\begin{array}{c}24.460 \\
37,5\end{array}$ \\
\hline Luxações/entorses & $\begin{array}{l}\text { № } \\
\%\end{array}$ & $\begin{array}{l}467 \\
2,8\end{array}$ & $\begin{array}{c}1.361 \\
4,2\end{array}$ & $\begin{array}{l}380 \\
3,9\end{array}$ & $\begin{array}{l}285 \\
4,5\end{array}$ & - & $\begin{array}{c}2.493 \\
3,8\end{array}$ \\
\hline L.intracran e int* & $\begin{array}{l}\text { № } \\
\%\end{array}$ & $\begin{array}{c}3.184 \\
19,4\end{array}$ & $\begin{array}{c}5.802 \\
17,9\end{array}$ & $\begin{array}{c}1.423 \\
14,7\end{array}$ & $\begin{array}{c}622 \\
9,8\end{array}$ & $\begin{array}{l}37 \\
8,9\end{array}$ & $\begin{array}{c}11.068 \\
16,9\end{array}$ \\
\hline Fer./l.tr.vasos** & $\begin{array}{l}\text { № } \\
\%\end{array}$ & $\begin{array}{c}2.088 \\
12,7\end{array}$ & $\begin{array}{c}5.843 \\
18,0\end{array}$ & $\begin{array}{l}1.896 \\
19,5\end{array}$ & $\begin{array}{c}1.356 \\
21,3\end{array}$ & $\begin{array}{l}16 \\
3,9\end{array}$ & $\begin{array}{c}11.199 \\
17,2\end{array}$ \\
\hline Corpos estranhos & $\begin{array}{l}\text { № } \\
\%\end{array}$ & $\begin{array}{l}189 \\
1,1\end{array}$ & $\begin{array}{l}121 \\
0,4\end{array}$ & $\begin{array}{l}46 \\
0,5\end{array}$ & $\begin{array}{l}19 \\
0,3\end{array}$ & - & $\begin{array}{l}375 \\
0,6\end{array}$ \\
\hline Queimaduras & $\begin{array}{l}\mathrm{N} N \\
\%\end{array}$ & $\begin{array}{c}1.797 \\
10,9\end{array}$ & $\begin{array}{c}1.267 \\
3,9\end{array}$ & $\begin{array}{l}282 \\
2,9\end{array}$ & $\begin{array}{l}117 \\
1,8\end{array}$ & $\begin{array}{c}2 \\
0,5\end{array}$ & $\begin{array}{c}3.465 \\
5,3\end{array}$ \\
\hline Envenenamentos & $\begin{array}{l}\text { № } \\
\%\end{array}$ & $\begin{array}{l}984 \\
6,0\end{array}$ & $\begin{array}{c}2.734 \\
8,4\end{array}$ & $\begin{array}{l}771 \\
7,9\end{array}$ & $\begin{array}{l}225 \\
3,6\end{array}$ & - & $\begin{array}{c}4.714 \\
7,2\end{array}$ \\
\hline Compl. cuid. méd. & $\begin{array}{l}\text { № } \\
\%\end{array}$ & $\begin{array}{c}226 \\
1,4\end{array}$ & $\begin{array}{c}1.344 \\
4,2\end{array}$ & $\begin{array}{l}550 \\
5,7\end{array}$ & $\begin{array}{l}280 \\
4,4\end{array}$ & $\begin{array}{c}7 \\
1,7\end{array}$ & $\begin{array}{c}2.407 \\
3,7\end{array}$ \\
\hline O utras lesões & $\begin{array}{l}\text { № } \\
\%\end{array}$ & $\begin{array}{l}969 \\
5,9\end{array}$ & $\begin{array}{c}2.305 \\
7,1\end{array}$ & $\begin{array}{l}714 \\
7,4\end{array}$ & $\begin{array}{l}397 \\
6,3\end{array}$ & $\begin{array}{c}1 \\
0,2\end{array}$ & $\begin{array}{c}4.386 \\
6,7\end{array}$ \\
\hline Efeitos tardios & $\begin{array}{l}\text { № } \\
\%\end{array}$ & $\begin{array}{l}109 \\
0,7\end{array}$ & $\begin{array}{l}230 \\
0,7\end{array}$ & $\begin{array}{l}96 \\
1,0\end{array}$ & $\begin{array}{l}58 \\
0,9\end{array}$ & $\begin{array}{l}241 \\
58,1\end{array}$ & $\begin{array}{l}734 \\
1,1\end{array}$ \\
\hline Total & $\begin{array}{l}\text { № } \\
\%\end{array}$ & $\begin{array}{c}16.447 \\
100,0\end{array}$ & $\begin{array}{c}32.386 \\
100,0\end{array}$ & $\begin{array}{l}9.700 \\
100,0\end{array}$ & $\begin{array}{l}6.353 \\
100,0\end{array}$ & $\begin{array}{c}415 \\
100,0\end{array}$ & $\begin{array}{c}65.301 \\
100,0\end{array}$ \\
\hline
\end{tabular}

* lesões intracranianas e internas

** ferimentos e lesões traumáticas dos vasos

zinhos perfazem $60,0 \%$ do total da faixa. Os envenenamentos mostraram-se pouco importantes para o grupo de 65 e mais anos.

A Tabela 7 mostra os tipos de lesão segundo os tempos de permanência (total em dias e média). Verifica-se que a maior média de permanência é apresentada pelo grupo dos "efeitos tardios das lesões" (15,5 dias), vindo, a seguir, as "queimaduras" (9,6 dias) e as "complicações de cuidados médicos e cirúrgicos" (7,0 dias). As "fraturas" ficaram em $4^{\circ}$ lu-

Tabela 7 - Hospitalizações por lesões e envenenamentos segundo tipo da lesão e tempo de permanência, Brasil, novembro de 1994.

\begin{tabular}{lrrr}
\hline Lesões & $\begin{array}{c}\text { Número } \\
\text { de Casos }\end{array}$ & $\begin{array}{c}\text { Permanência } \\
\text { Total } \\
\text { (dias) }\end{array}$ & $\begin{array}{c}\text { Média } \\
\text { (dias) }\end{array}$ \\
\hline Fraturas & 24.460 & 129.617 & 5,3 \\
Luxações/entorses & 2.493 & 10.799 & 4,3 \\
L. intracran. e int. & 11.068 & 52.783 & 4,8 \\
Fer./l. traum. vasos & 11.199 & 52.947 & 4,7 \\
Corpos estranhos & 375 & 771 & 2,1 \\
Queimaduras & 3.465 & 33.099 & 9,6 \\
Envenenamentos & 4.714 & 14.966 & 3,2 \\
Compl. cuid. méd. & 2.407 & 16.861 & 7,0 \\
Outras lesões & 4.386 & 22.850 & 5,2 \\
Efeitos tardios & 734 & 11.401 & 15,5 \\
\hline Total & 65.301 & 346.094 & 5,3 \\
\hline
\end{tabular}

gar com 5,3 dias em média. Dados dos Estados Unidos evidenciam que, naquele país, em 1991, a média de permanência por todas as lesões foi de 6,9 dias, sendo que para as fraturas foi de 8,4 dias, com destaque para as de colo de fêmur, que foi de 12,3 dias (NCHS $\left.{ }^{11}, 1993\right)$.

Relativamente à distribuição dos óbitos hospitalares segundo sexo e idade (Tabela 8), verifica-se uma concentração no sexo masculino $(71,7 \%$ contra $28,3 \%$ no feminino), onde as taxas são também mais elevadas. Nos grupos etários essa predominância somente não ocorreu nos menores de 15 anos, nos quais a taxa de mortalidade hospitalar foi maior entre as mulheres. Chama a atenção o valor crescente dessas taxas quanto mais idosa for a população internada. No grupo de 65 anos e mais ela é, praticamente, igual a três vezes a TMH média nos homens e duas vezes a TMH média das mulheres (respectivamente 6,5 e 4,2 por cem internações do sexo e da idade). As taxas nos sexos masculino e feminino, no Estado de São Paulo, eram, em 1980, respectivamente 2,3 e $0,9 \%$, sendo igual a $1,8 \%$ para os dois sexos (Ferreira e Faria ${ }^{2}$, 1986), valor igual ao dos Estados Unidos, em $1993\left(\mathrm{NCH}^{12}, 1995\right)$.

A Tabela 9 mostra que o valor médio da TMH no Brasil foi de 2,2\%, o que significa um aumento de mais de $20 \%$ em relação à taxa americana para 1993 , $\left(\mathrm{NCHS}^{12}, 1995\right)$ que foi de $1,8 \%$ para as "lesões e 
Tabela 8 - Taxa de mortalidade hospitalar (TMH)* por "lesões e envenenamentos" segundo sexo e idade, Brasil, novembro de 1994.

\begin{tabular}{|c|c|c|c|c|c|c|}
\hline \multirow[b]{2}{*}{ Idade (em anos) } & \multicolumn{2}{|c|}{ Masculino } & \multicolumn{2}{|c|}{ Feminino } & \multicolumn{2}{|c|}{ Total } \\
\hline & $\mathrm{N} 0$ & $\mathrm{TMH}$ & No & $\mathrm{TMH}$ & $\mathrm{N} \cong$ & $\overline{\mathrm{TMH}}$ \\
\hline$<15$ & 92 & 0,8 & 69 & 1,1 & 161 & 0,9 \\
\hline 15 a 44 & 670 & 2,5 & 152 & 1,6 & 822 & 2,2 \\
\hline 45 a 64 & 231 & 3,3 & 89 & 2,3 & 320 & 2,9 \\
\hline $65 e+$ & 199 & 6,5 & 160 & 4,2 & 359 & 5,2 \\
\hline Total & 1.192 & 2,4 & 470 & 2,0 & 1.662 & 2,2 \\
\hline
\end{tabular}

* Taxa de mortalidade hospitalar (por 100 internações).

Tabela 9 - Ó bitos hospitalares por "lesões e envenenamentos" segundo o tipo de lesão e o momento da sua ocorrência, Brasil, novembro de 1994.

\begin{tabular}{|c|c|c|c|c|c|c|c|}
\hline \multirow[b]{2}{*}{ Lesões } & \multicolumn{2}{|c|}{$<48 \mathrm{~h}$} & \multicolumn{2}{|c|}{$48 \mathrm{he}+$} & \multicolumn{2}{|c|}{ Total } & \multirow{2}{*}{$\mathrm{TMH}$} \\
\hline & No & $\%$ & $\mathrm{~N} 0$ & $\%$ & $\mathrm{~N} N$ & $\%$ & \\
\hline Fraturas & 52 & 24,3 & 162 & 75,7 & 214 & 100,0 & 0,9 \\
\hline Luxações/entorses & 6 & 28,6 & 15 & 71,4 & 21 & 100,0 & 0,8 \\
\hline L. intracan. e int* & 269 & 40,9 & 389 & 58,1 & 658 & 100,0 & 6,0 \\
\hline Fer./l. tr. vasos** & 49 & 26,5 & 136 & 73,5 & 185 & 100,0 & 1,7 \\
\hline Corpos estranhos & - & - & -- & - & - & - & \\
\hline Queimaduras & 16 & 16,8 & 79 & 83,2 & 95 & 100,0 & 2,7 \\
\hline Envenenamentos & 41 & 47,1 & 46 & 52,9 & 87 & 100,0 & 1,8 \\
\hline Compl.cuid. méd. & 15 & 25,4 & 44 & 74,6 & 59 & 100,0 & 2,5 \\
\hline O utras lesões & 58 & 45,7 & 69 & 54,3 & 127 & 100,0 & 2,9 \\
\hline Efeitos tardios & 1 & 16,7 & 5 & 83,3 & 6 & 100,0 & 0,8 \\
\hline Total & 507 & 34,9 & 945 & 65,1 & 1.452 & 100,0 & 2,2 \\
\hline
\end{tabular}

Nota: Em 210 óbitos não foi possível determinar o tipo de lesão.

* lesões intracranianas internas

** ferimentos e lesões traumáticas dos vasos

TMH - Taxa de Mortalidade Hospitalar.

envenenamentos". Esses valores variaram desde zero, correspondendo a nenhuma morte no grupo de "efeitos da ingestão de corpos estranhos" até $6 \%$ das internações, no caso das "lesões intracranianas". Essas lesões têm valor bastante distanciado em relação às demais causas, o que é fácil de ser entendido, dada a sua gravidade. Esse fato é reforçado pela análise do "momento" da ocorrência desses óbitos: 40,9\% deles verificam-se nas primeiras 48 horas de internação, contra valores mais baixos encontrados em outros tipos de lesão, como por exemplo as "queimaduras $(16,8 \%)$, os "efeitos tardios" $(16,7 \%)$ e, até mesmo, as "fraturas" e os "ferimentos", onde a morte é também mais tardia.

\section{CONSIDERAÇÕ ES FIN AIS}

Apesar das críticas comumente feitas às estatíticas hospitalares, pela sua seletividade ou mesmo pela sua qualidade, seria desejável sua utilização, seja com finalidades epidemiológicas ou administrativas. No entanto, no Brasil, essas informações, geradas a partir das AIHs, são acrescidas de novos problemas, uma vez que este é um instrumento financeiro, criado com o objetivo de pagamento dos serviços prestados pela rede hospitalar e, desta forma, a serviço muito mais do faturamento do que de outras questões.

Porém, quando se está frente ao volume de internações do País ou, mesmo, de algumas regiões do País, possível pelos novos meios de disseminação dos dados, acredita-se que algumas das falhas dessa fonte possam estar minimizadas ou diluídas, dando oportunidade a que se aproveitem essas informações, se não do ponto de vista epidemiológico, pelo menos administrativamente. Verifica-se que os números permitem visualizar uma tendência, ou um padrão, e mostram coerência, corroborando essa idéia.

A partir dos resultados do presente trabalho, seria muito oportuno poder fazer recomendações de prevenção ou trazer propostas de ações, não fosse o fato, lamentável, de os hospitais não colocarem, nos casos de lesões e envenenamentos, a causa externa daquela violência, ou seja, como ocorreram todos os ferimen- 
tos, fraturas e queimaduras. Das mais de 70.000 hospitalizações por lesões e envenenamentos estudadas, apenas 500 apresentavam diagnóstico secundário aproveitável. No restante dos casos, quando havia, ele se resumia a repetir o diagnóstico principal.

Diante do elevado número de internações ocorridas no País (cerca de 14 milhões/ano), pode-se perceber a perda de informação em relação às lesões e envenenamentos que correspondem a $6 \%$ das hospitalizações, e que acontecem, principalmente, entre os adultos jovens.

\section{REFERÊN CIAS BIBLIO G RÁFICAS}

1. COM-RUELLE, L. Les étapes diagnostiques et la maladie principale des hospitalisés en 1992. Paris, Centre de Recherche d'étude et Documentation en Economie de la Santé, 1995.

2. FERREIRA, J.M.N. \& FARIA, C.G. Morbidade hospitalar no Estado de São Paulo. Rev. Fund. SEADE/S. Paulo em Perspec., 2 : 31-52, 1986.

3. FUNDAÇÃO IBGE. Censo demográfico de 1970. Rio de Janeiro, 1973.

4. FUNDAÇÃO IBGE. Censo demográfico de 1980. Rio de Janeiro, 1982.

5. FUNDAÇÃO IBGE. Censo demográfico de 1991. Rio de Janeiro, 1993.

6. LEBRÃO, M.L. Evolução da morbidade hospitalar -Vale do Paraíba, 1975 e 1988, São Paulo, 1994 [Tese de LivreDocência Faculdade de Saúde Pública da USP]

7. LEVCOVITZ, E. \& PEREIRA, T.R.C. SIH/SUS (Sistema AIH): uma análise do sistema público de remuneração de internações hospitalares no Brasil -1983-1991: Rio de Janeiro, UERJ/Instituto de Medicina Social, 1993.

8. MELLO JORGE, M.H.P. Investigação sobre a mortalidade por acidentes na infância. São Paulo, 1988. [Tese de LivreDocência Faculdade de Saúde Pública da USP].

9. MINISTÉRIO DA SAÚDE/FNS/CENEPI. Inf. Epidemiol. SUS , 1 (1), 1992.

10. MINISTÉRIO DA SAÚDE/DATASUS. Internações hospitalares: subsídios para análise, 1994. Rio de Janeiro, 1995.
Dessa forma, como conclusão, poder-se-ia enfatizar a importância de os hospitais apresentarem, também, a causa externa das lesões e, não somente a natureza dessas lesões. Não se previne a fratura, a queimadura ou o traumatismo crânio-encefálico mas, sim, a queda; o acidente com fogo ou o acidente automobilístico.

Da maneira como esses dados estão postos, resta apenas o aproveitamento dos mesmos para o pagamento e, na melhor das hipóteses, para o planejamento e administração dos serviços hospitalares.

11. NATIONAL CENTER FOR HEALTH STATISTICS.

National hospital discharge survey: annual sumary 1993. Vital Health Stat. Sér., 13 (114) 1993.

12. NATIONAL CENTER FOR HEALTH STATISTICS. National hospital discharge survey: annual sumary 1993. Vital Health Stat. Sér, 13 (121) 1995.

13. NORDIC MEDICO-STATISTICAL COMMITTEE (NOMESCO). Health statistics in the Nordic Countries 1990. Copenhagem, 1992.

14. ORGANIZAÇÃO MUNDIAL DA SAÚDE. Manual da classificação estatística internacional de doenças, lesões e causas de óbitos; $9^{a}$ rev.1975. São Paulo, Centro da OMS para Classificação de Doenças em Português, 1980.

15. PAIVA, T.M. Estudo da morbidade hospitalar: Hospital Municipal Dr. Carmeno Caricchio, outubro e dezembro de 1994. São Paulo, 1995. [mimeografado].

16. POKRAS, R. et al. Trends in hospital utilization:United States, 1965-86 Hyattsville, MD. National Center for Health Statistics, Vital Health Stat. Sér., 13 (101) 1989.

17. SANTO, A.H. Causas múltiplas de morte: formas de apresentação e métodos de análise. São Paulo, 1988 [Tese de Doutorado-Faculdade de Saúde Pública da USP].

18. VERAS, C.M.T. Equity in the use of private hospitals by a compulsory insurance in the city of Rio de Janeiro, Brasil in 1986. London, 1992. [Tese de Doutorado - London School of Economics and Political Sciences].

19. YAZLLE ROCHA, J.S. Padrões de morbidade hospitalar: considerações para uma política de assistência médicohospitalar. Rev. Saúde Pública., 14: 58-64, 1980 